\title{
Time-Domain Joint Estimation of Fine Symbol Timing Offset and Integer Carrier Frequency Offset
}

\author{
Tae-Hwan Kim and In-Cheol Park \\ Department of Electrical Engineering \\ Korea Advanced Institute of Science and Technology (KAIST) \\ 373-1 Guseong-dong, Yuseong-gu, Daejeon 305-701, Republic of Korea \\ \{thkim@eeinfo.kaist.ac.kr, icpark@ee.kaist.ac.kr\}
}

\begin{abstract}
In this paper, we propose an efficient synchronization method to jointly estimate fine symbol timing offset (STO) and integer carrier frequency offset (CFO). The proposed method is to perform cross-correlation between received samples and pre-rotated training sequences. Experimental results on IEEE 802.16d systems show that the proposed method is significantly superior to the previous approaches in both estimations. Since the fine STO and the integer CFO are jointly estimated in the time domain in an onthe-fly manner, the proposed method requires no additional buffers. Moreover, the proposed joint estimation has an effect of eliminating the processing ordering dependency of the two estimations, being attractive for systems requiring tight synchronization.
\end{abstract}

\section{INTRODUCTION}

Orthogonal frequency division multiplexing (OFDM) is a parallel transmission technique in which all the sub-channels are modulated with orthogonal bases by using the discrete Fourier transform (DFT) [1], [2]. OFDM presents an effective solution for the frequency selectivity problem mainly caused by multi-path fading, and mitigates inter-symbol interferences by allocating a relatively low data rate to each sub-channel and providing a cyclic prefix (CP) for each symbol. As subchannel bands are overlapping one another to achieve a fairly good spectral efficiency in OFDM systems, the performance is very sensitive to sub-channel interferences caused by the frequency offset [3]. Additionally, the timing offset larger than the CP leads to inter-symbol interference, degrading the system performance severely. Therefore, the advantages of OFDM systems can be achieved only with good synchronizations both in time and frequency.

A general synchronization method widely used in burstmode OFDM systems is to calculate auto-correlation between received samples [4]-[8]. Though this is robust to noise and the carrier frequency offset (CFO), the accuracy of the symbol timing offset (STO) estimation is very low, and the CFO estimation range is limited. Since the method is insufficient to meet the tight synchronization specification demanded for such systems as IEEE 802.16d, one of the WiMAX standard [9], it is usually required to estimate the fine STO and the integer CFO being out of the estimation range [7], [8].

Targeting IEEE $802.16 \mathrm{~d}$ systems, we propose a new approach to jointly estimate the two offsets in the time domain. The proposed method is to perform cross-correlations in parallel with pre-rotated training sequences. Since the two estimations are performed jointly in the time domain, one estimation does not depend on the other estimation. In addition, an on-the-fly computation scheme is proposed to reduce the overall processing latency without employing additional buffers.

The rest of the paper is organized as follows: Section II and Section III describe the definition of the estimation problems and review previous works. In Section IV, the joint estimation method is proposed based on the analysis of the CFO. Experimental results are presented in Section $\mathrm{V}$ and conclusions are drawn in Section VI.

\section{Fine STO Estimation}

The objective of fine STO estimation is to detect the starting point of a DFT window, which is needed for OFDM demodulation in the receiver. The downlink preamble structure of IEEE 802.16d system is shown in Fig. 1 [9]. As the two long training sequences in the downlink preamble are usually demodulated to estimate the fading factors of the channel, the fine STO estimation should be completed prior to the long training sequences to detect the boundary.

If the STO estimation error is smaller than the CP length, it can be interpreted in the time domain as a cyclic shift of the symbol. In the frequency domain, this cyclic shift has an effect of rotating the spectral components by the amount of its frequency domain index, and can be compensated by equalizing the channels. When the estimated starting point is too early or too late compared to the guard interval of the $\mathrm{CP}$ length, the performance is significantly degraded due to intersymbol interference.

A simple method to estimate the fine STO is to detect the last boundary of the short training sequences by perceiving the falling edge of the auto-correlation curve. This method is robust to the $\mathrm{CFO}$ and leads to a simple hardware implementation. As the coarse STO estimation is usually based on auto-correlation [4]-[8], a hardware structure similar to that used for the coarse STO estimation can be applied here. Besides, it is not complex if the recursive auto-correlation that

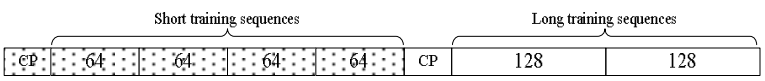

Figure 1. Downlink preamble structure of IEEE 802.16d 
can be implemented with only two complex multipliers and an accumulator is employed [4]. However, it is almost impossible to apply this method to such a system that demands high estimation accuracy. In IEEE 802.16d, for example, the allowed STO ranges from -4 to +4 samples, which is too tight for this method to meet. There have been some works that enhance the accuracy by employing other timing metrics as in [6], but such an approach increases computational complexity considerably.

To achieve more accurate estimation, cross-correlations between received samples and training sequences have been tried in [7], [8], [11], [14]. The cross-correlation is expressed as

$$
X_{n}=\sum_{i=1}^{W} r_{n-i} s_{n-i}^{*},
$$

where $r_{\mathrm{n}}$ is the $n$-th received sample, $s_{n}$ is the $n$-th sample of the training sequence in the preamble, and $W$ is the length of the training sequence that is either 64 or 128 in the downlink preamble of IEEE 802.16d [9]. When $r_{n}$ and $s_{n}$ are matched together, the cross-correlation, $X_{n}$, has a peak value. We can estimate the boundary of the short training sequences by detecting the peak. This method can achieve high estimation accuracy, and can be successful even in a very low SNR. Additionally, the cross-correlation can be performed with a very small bit-width [14], which enables $W$ complex multiplications and their summation in (1) to be calculated in a clock period affordable in a practical implementation. This method is, however, much vulnerable to the CFO. If this method is applied to the samples contaminated by some CFOs, it can result in severe performance degradation, as the peak is no longer prominent.

\section{INTEGER CFO ESTIMATION}

The CFO means the carrier frequency mismatch between the transmitter and the receiver, which is usually induced by the impairments of the RF front-end and the complicated effects in the transmission channels such as Doppler spread. The CFO estimation plays an important role in OFDM systems, as it has a significant effect on the orthogonality of sub-channels. To represent the interfering effect, the CFO is usually normalized by the sub-carrier spacing. The integer component of the CFO represents a cyclic shift in the frequency-domain symbol, while the fractional component causes interferences between sub-channels.

For the fractional CFO estimation, the average phase difference between two samples corresponding to the same position in the training sequence is measured by performing auto-correlation as follows.

$$
\frac{1}{W} \tan ^{-1}\left(\sum_{i=1}^{W} r_{n-i}^{*} \cdot r_{n-i-W}\right)
$$

This has a problem that the estimation range is limited. In IEEE $802.16 \mathrm{~d}$, for instance, its range is up to \pm 2 sub-carrier spacing, whereas the CFO can be larger than this range.
The integer CFO is commonly estimated in the frequency domain by performing cross-correlation [7], [10], [12]. As this offset causes a cyclic shift in the frequency domain, the crosscorrelation between the training sequence and the cyclic shifted symbol is performed in the frequency domain. This can be expressed as follows,

$$
\underset{d}{\arg \max }\left|\sum_{k=1}^{N} S_{k}^{*} R_{(k+d) \% N}\right|,
$$

where $R_{k}$ and $S_{k}$ are the $k$-th frequency components of the received symbol and the training sequence in the frequency domain, respectively. The symbol size $N$ is equal to the DFT size, and $\%$ represents the modulo operation. The integer CFO is estimated by searching for a shift amount, $d$, that yields a peak in the cross-correlation as expressed in (3). The shift amount, $d$, is usually a multiple of the estimation range of the fractional $\mathrm{CFO}$, because only the integer $\mathrm{CFO}$ remains once the fractional CFO is corrected. Though this scheme is intuitive, it needs almost perfect STO estimation to achieve a reasonable performance [12]. To work-around this problem, the cross-correlation is modified with the concept of the coherence phase bandwidth so as to estimate the integer CFO even in the presence of moderate STO [12].

Moreover, the integer CFO estimation should be performed before the channel estimation in the frequency domain. The training sequences in the preamble are usually used in the frequency domain to acquire the channel information, especially in burst-mode OFDM systems such as IEEE 802.16d [9]. Thus the integer CFO estimation should be done in an early processing stage.

\section{JOINT ESTIMATION OF Fine STO AND INTEGER CFO}

A new method that can jointly estimate the fine STO and the integer CFO in the time domain is proposed in this section. The proposed method is based on the parallel crosscorrelations with pre-rotated training sequences, and is free from the problem that the performance of one estimation is closely related to the error of the other estimation. Additionally, since both estimation results are available at the same time, no extra buffers are required for the integer CFO estimation.

\section{A. Analysis of Integer $\mathrm{CFO}$}

The fractional CFO can be corrected before the fine STO estimation by computing the auto-correlation jointly with the coarse STO estimation [7], [8]. Thus, we assume that there remains only the integer $\mathrm{CFO}$ in the received samples when the fine STO is estimated. The fractional CFO can be estimated by measuring the phase difference between the two samples corresponding to the same position in the training sequence. As the received samples are rotated in proportion to the CFO, they can be represented as the phasors as shown in Fig. 2(a), where the received samples, $r_{n}$ and $r_{n-W}$ are complex values and $W$ is the length of the training sequence. Since the two samples correspond to the same position of the training 


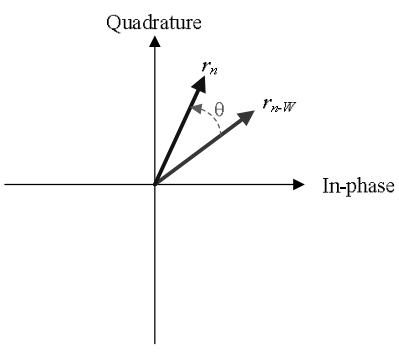

(a) No revolution

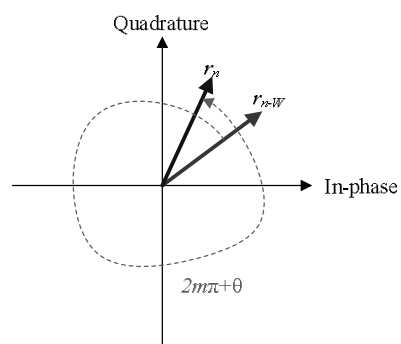

(b) $m$ revolutions
Figure 2. Phasor representation of CFO

sequence, they were equal to each other at the transmitter. The rotation of $r_{n-W}$ is expressed as

$$
r_{n}=e^{j \theta} r_{n-W}
$$

where $\theta$ is the phase difference between the two samples. Based on this relation, we can estimate the CFO as $\theta / W$. For more reliable estimation, the $\mathrm{CFO}$ is estimated by taking an average for many samples as in (2).

The rotation in Fig. 2(a) can be generalized as shown in Fig. 2(b). In this case, $r_{n}$ is rotated by more than one revolution as follows,

$$
r_{n}=e^{j 2 m \pi} e^{j \theta} r_{n-W},
$$

where $\mathrm{m}$ is the number of revolutions. We can recognize only the phase difference $\theta$ in the range of $[0,2 \pi)$, while the actual phase difference is $2 m \pi+\theta$. As the actual phase difference, $2 m \pi+\theta$, cannot be distinguished from $\theta$, the CFO is still estimated as $\theta / W$. Therefore, the residual $\mathrm{CFO}, 2 m \pi / W$, remains in the samples even after the fractional $\mathrm{CFO}, \theta / W$, is corrected. The rotation due to this residual component is represented as

$$
e^{j 2 m \pi / W}=\omega_{W}^{-m}=\omega_{N}^{-k m},
$$

where $N$, expressed as $W$ times $k$, is the number of the DFT points and $\omega_{N}$ is the unit twiddle factor, $\exp (-j 2 \pi / N)$. As $N=$ 256 and $W=64$ or 128 in the target system [9], $k$ is either 4 or 2 , respectively. In (6), the remaining term is an integer multiple of the sub-carrier spacing, and can be regarded as an integer power of the twiddle factor, causing a cyclic shift of the symbol in the frequency domain as indicated by the shifting property of the DFT. Fig. 3 illustrates the integer CFO remaining in the received samples, where a CFO of +5.5 subcarrier spacing is exemplified with assuming that the fractional $\mathrm{CFO}$ range estimated by the short training sequence is from -2 to +2 sub-carrier spacing. In this case, the estimated fractional $\mathrm{CFO}$ is +1.5 sub-carrier spacing and the residual integer $\mathrm{CFO}$ is +4 sub-carrier spacing. If the fractional $\mathrm{CFO}$ is corrected in the preceding steps, the samples to be used for the fine STO estimation have only a CFO that is an integer multiple of the estimation range of the fractional CFO. This

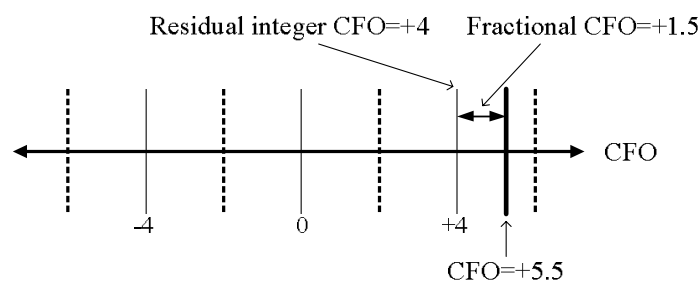

Figure 3. $\mathrm{CFO}$ Estimation $(\mathrm{CFO}=5.5$ sub-carrier spacing)

assumption is usually valid in the synchronizer of the burstmode OFDM receivers [7], [8].

\section{B. Cross-correlation with the Pre-rotated Sequences}

To exploit the discreteness of the residual integer CFO, we propose a new estimation scheme. Since the worst CFO in a profile of IEEE $802.16 \mathrm{~d}$ is less than \pm 6 sub-carrier spacing [9], the residual integer $\mathrm{CFO}$ can be 0 or \pm 4 sub-carrier spacing if the fractional $\mathrm{CFO}$ is first corrected using the short training sequences of length 64. If the cross-correlation is performed for the fine STO estimation, the residual integer CFO degrades its performance severely.

As the integer CFO is limited to three cases, we can prepare three pre-rotated training sequences each of which is rotated by one of the possible integer CFOs. The pre-rotated training sequences are involved in the computation of cross-correlation. These pre-rotated training sequences can be expressed as

$$
s_{n, p}=s_{n} \cdot \omega_{N}^{p n},
$$

where $p$ is 0 or $\pm 4, s_{n}$ is the $n$-th sample in the training sequence, $s_{n, p}$ is the $n$-th sample in the training sequence rotated by an integer CFO of $p$. Let $\check{r}_{n}$ be the sample of which fractional $\mathrm{CFO}$ is corrected.

As the residual CFO of $\check{r}_{n}$ is 0 or \pm 4 sub-carrier spacing, $\check{r}_{n}$ must be matched with one of the pre-rotated sequences, $s_{n, 0}$, $s_{n,+4}$ or $s_{n,-4}$. The matched one will have a large peak. If the peak occurs at $n$ and $p$, then the fine STO and the integer CFO correspond to $n$ and $p$, respectively. We can formalize this observation as

$$
(n, p)=\arg \max _{(n, p)}\left|X_{n, p}\right|
$$

where $X_{n, p}$ denotes the cross-correlation between $\check{r}_{n}$ and $s_{n, p}$.

For example, the proposed estimation is simulated with a CFO of +5.5 sub-carrier spacing in a multi-path fading environment in which the channel parameters conform to those of SUI-3 channel and SNR is $2 \mathrm{~dB}$ [13]. As shown in Fig. 4 , where $X_{n, p}$ is plotted separately for each $p$, the peak occurs at $n=25$ and $p=-4$, implying that the fine STO is +25 and the integer $\mathrm{CFO}$ is -4 sub-carrier spacing.

Fig. 5 shows the hardware architecture of the proposed method, where $\check{r}_{n}$ is cross-correlated with the pre-rotated training sequences. This architecture can be thought as a combination of three FIR filters whose length is 64 . The direct 


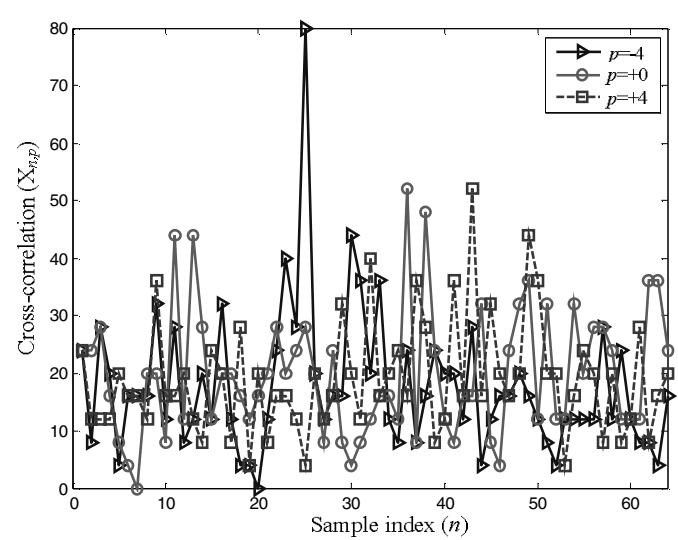

Figure 4. Parallel cross-correlations for the joint estimation

form is suitable for the hardware implementation because the total number of buffers is less than that of the transposed form and the cross-correlation does not need to operate at a high clock frequency in the target system.

As indicated in (8), we have to search $64 \times 3$ points to find the peak. It is important to reduce the complexity of the two dimensional search. First of all, the two-dimensional search is converted to two one-dimensional searches. For each sample, three cross-correlations are calculated, but only one that is associated with the maximum value is considered as a candidate for the peak. Then the peak is chosen among these candidates. As depicted in Fig. 5, this structure is simple and easy to implement. Second, the cross-correlation can be calculated with the sign-quantized samples as shown in Fig 5. In the figure, $\operatorname{sign}(\cdot)$ means the MSB of the 2's complement number. Thus only one bit is involved in the computation of the cross-correlation, reducing the complex multiplication in the cross-correlation to only a few gates in the implementation. In addition, multiplying a sign-quantized sample by the prerotated training sequence results in either \pm 2 or $\pm j 2$. As it is sufficient to detect the peak relatively rather than absolutely, all the product results can be scaled down without any loss of accuracy, meaning that only \pm 1 or $\pm j$ is produced in each tap

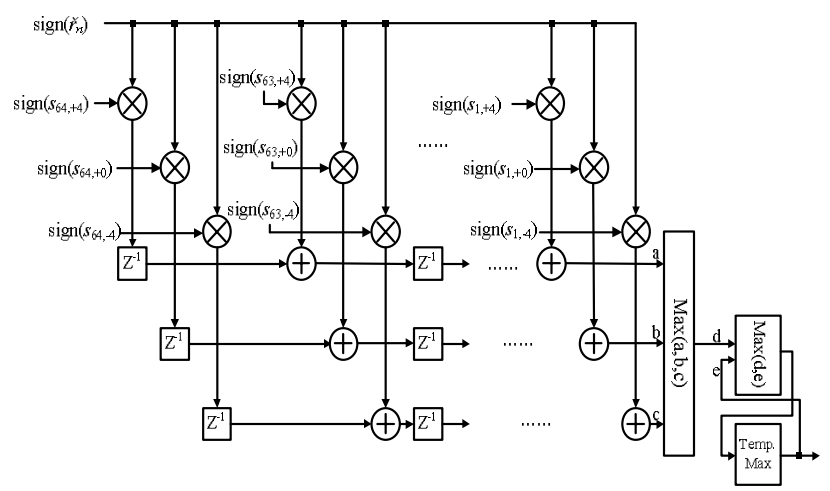

Figure 5. Proposed architecture for the joint estimation of the filter structure shown in Fig. 5. Thus the filter output can be represented in at most 7 bits, as the filter output is generated by summing up to 64 products. With a little performance degradation, moreover, the absolute operation can be approximated based on the following equation.

$$
|x+j y| \approx|x|+|y|,
$$

where $x$ and $y$ are real numbers. Therefore, the proposed method can be efficiently implemented without increasing the hardware complexity considerably. Finally, the proposed joint estimation is active only for 64 sample clocks per frame. Even if the power consumption might be increased a little due to the parallel processing, its effect on the overall power consumption of the target system is negligible due to the short active time.

\section{PERFORMANCE RESULTS}

In this section, we evaluate the performance of the proposed scheme. The simulation environment is the multi-path fading channel in which the channel parameters are conformed to those in SUI-3 channel, CFO is +5.5 sub-carrier spacing, and the whole SNR range specified in IEEE $802.16 \mathrm{~d}$ is taken into account [9], [13].

Fig. 6 shows the simulation result of the fine STO estimation, where errors are presented in an RMS manner. The STO should be lower than 4 in the target system [9]. Compared to the conventional method based on autocorrelation, the proposed method shows an excellent performance in the fine STO estimation for every SNR simulated and meets the performance requirement for the whole SNR range, while the conventional one does not. The auto-correlation-based method is used in the comparison, because this estimation should be robust to the residual integer CFO. The previous method based on a single cross-correlation is not appropriate because its sensitivity to the residual integer $\mathrm{CFO}$ is severe.

Fig. 7 shows the simulation result of the integer CFO estimation, which compares the result of the proposed method with those of two previous ones. One performs the

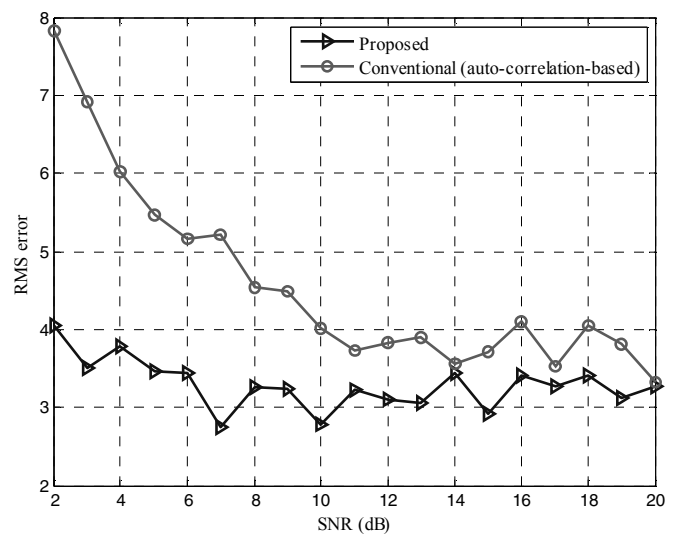

Figure 6. Simulation result of the fine STO estimation 


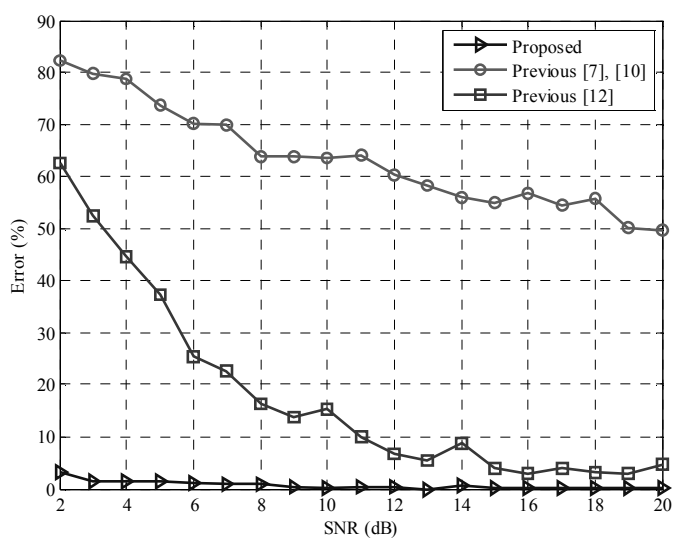

Figure 7. Simulation result of the integer CFO estimation

conventional cross-correlation in the frequency domain with the cyclic-shifted sequences, as defined in (3) [7], [10], and the other performs the cross-correlation modified with the concept of the coherence phase bandwidth proposed in [12]. To achieve robustness to the CFO, both of the previous schemes assume that the fine STO was accurately estimated by performing auto-correlation. The performance of the proposed method is excellent even in a very low SNR. Additionally, the proposed method can estimate the integer CFO in the time domain jointly with the fine STO, while the previous methods have to estimate them separately and cannot estimate the integer CFO until the symbol is transformed to the frequency domain. This means that the proposed method can reduce overall processing latency and buffers required to store samples.

\section{CONCLUSIONS}

We have proposed an efficient synchronization method for burst-mode OFDM systems such as IEEE 802.16d. In the proposed method, the fine STO and the integer CFO are jointly estimated in the time domain by performing crosscorrelation between received samples and pre-rotated training sequences. Simulation results show that the proposed approach is much superior to the previous ones in both estimations. Since the fine STO and the integer CFO are jointly estimated in the time domain in an on-the-fly manner, the proposed approach requires no additional buffers. In addition, the proposed joint estimation has an effect of eliminating the dependency of the processing order of the two estimations, whereas the ordering dependency is inevitable in the previous approaches estimating them separately.

\section{REFERENCES}

[1] Saltzberg. B., "Performance of an efficient parallel data transmission System," IEEE Trans. on Communications, vol.15, issue.6, pp805-811, Dec 1967.

[2] Weinstein.S., Ebert.P., "Data transmission by frequency-division multiplexing using the discrete Fourier transform," IEEE Trans. on Communications, vol.19, issue.5, pp628-634, Oct 1971.

[3] Pollet. T, Van Vladel. M. and Moeneclaey. M., "BER sensitivity of OFDM systems to carrier frequency offset and Wiener phase noise," IEEE Trans. on Communication, vol.43, issue.234, pp191-193, Feb/Mar/Apr 1995.

[4] Schmidl. T.M. and Cox, D.C, "Robust frequency and timing synchronization for OFDM," IEEE Trans. on Communications, vol.45, issue.12, pp1613-1621, Dec 1997.

[5] Fort.A. and Eberle.W., "Synchronization and AGC proposal for IEEE 802.11a burst OFDM systems," in Proc. of Global Telecommunications Conference 2003, vol.3, pp1335-1338.

[6] Ch. Nanda Kishore and V. Umapathi Reddy, "A frame synchronization and frequency offset estimation algorithm for OFDM system and its analysis," EURASIP Journal on Wireless Communications and Networking, vol. 2006, Article ID 57018, 2006.

[7] Mody, A.N. and Stüber, G.L., "Receiver implementation for a MIMO OFDM system," in Proc. of Global Telecommunications Conference 2002, vol.1, pp716-720.

[8] Krstic, M., Troya, A., Maharatna, K. and Grass, E., "Optimized lowpower synchronizer design for the IEEE 802.11a standard," in Proc. of ICASSP 2003, vol.2, ppII-333-6

[9] IEEE Std. 802.16. IEEE Standard for Local and Metropolitan Area Networks Part 16: Air Interface for Fixed Broadband Wireless Access Systems, 2004.

[10] H.Nogami and T. Nagashima, "A frequency and timing period acquisition technique for OFDM Systems," in Proc. of PIRMC 1995, vol.3, pp1010-1015.

[11] Tufvesson F., Edfors O., and Faulkner, M., "Time and frequency synchronization for OFDM using PN-sequence preambles," in Proc. of Vehicular Technology Conference 1999, vol.4, pp2203-2207.

[12] Keukjoon Bang and Namshin Cho et al., "A coarse frequency offset estimation in an OFDM system using the concept of the coherence phase bandwidth," IEEE Trans. on Communication, vol.49, issue.8, pp13201324, Aug 2001.

[13] V.Erceg, K.V.S Hari, M.S. Smith et al., "Channel models for fixed wireless applications," Contribution IEEE 802.16.3c-01/29r1, Feb. 2001.

[14] Kun-Wah Yip, Yik-Chung Wu, and Tung-Sang Ng, "Design of multiplierless correlators for timing synchronization in IEEE 802.11a Wireless LANs," IEEE Trans. on Consumer Electronics, vol.49, issue.1, pp107-114,Feb.2003. 